PHYSICAL REVIEW D 95, 039904(E) (2017)

\title{
Erratum: Dimension-seven operators in the standard model \\ with right handed neutrinos \\ [Phys. Rev. D 94, 055022 (2016)]
}

Subhaditya Bhattacharya and José Wudka

(Received 8 February 2017; published 17 February 2017)

DOI: 10.1103/PhysRevD.95.039904

Except when noted otherwise, all operators are assumed to be family diagonal; if this is relaxed additional operators may appear. 